\title{
Vasoplegic syndrome after oral nimodipine application in patients with subarachnoid hemorrhage
}

\begin{abstract}
Subarachnoid hemorrhage remains a serious disease with high mortality rate and often disastrous neurological outcome. After improvement of techniques to secure the underlying aneurysm leading to decrease of immediate complications such as rebleeding, cerebral vasospasm remains the major cause for mortality and morbidity after subarachnoid hemorrhage. The only FDA approved drug for treatment of cerebral vasospasm is the calcium antagonist Nimodipine that has shown beneficial effects on outcome. It is safe, cost efficient and the most widely studied drug for treatment of cerebral vasospasm. But it has reported side effects such as systemic hypotension, especially when used intravenously. The present paper reports about the occurrence of severe systemic catecholamine refractory hypotension after oral application of the standard dosage of $60 \mathrm{mg}$ nimodipine. In those patients we were only able to establish a sufficient arterial blood pressure after application of methylene blue suggesting that at least part of the underlying mechanism was NO related vasoplegia. Keeping in mind that vasoplegia can occur even after oral nimodipine application we suggest that there should be a test dosage of 15-30 mg nimodipine applied to evaluate the impact on each patient and avoid potential lethal hypotension.
\end{abstract}

Keywords: vasoplegic syndrome oral nimodipine, subarachnoid hemorrhage, methylene blue application
Volume I Issue 6 - 2014

\author{
Bele S,' Scheitzach J,' Kieninger M,'² \\ Hochreiter A,' Schödel P,' Bründl E, \\ Schebesch K-M,' Brawanksi A' \\ 'Department of Neurosurgery, Regensburg University, Germany \\ ${ }^{2}$ Department of Anaesthesiology, Regensburg University, \\ Germany
}

\author{
Correspondence: Sylvia Bele, Department of Neurosurgery, \\ Regensburg University, Franz Josef Strauß Allee II, 93052 \\ Regensburg, Germany, Tel 49941944907I, \\ Email Sylvia.bele@ukr.de
}

Received: November 08, 20I4 | Published: December 02, 2014
Abbreviations: WFNS, world federation of neurological surgeons scale for severity of sah; MCA, middle cerebral artery; ICA, internal carotid artery; $\mathrm{ACoA}$, anterior communicating artery; PCoA, posterior communicating artery; M, male; F, female; MAP1, mean arterial blood pressure before methylene blue application; MAP2, mean arterial blood pressure after methylene blue application; Medication, means anti hypertensive medication prior to admission; GOS, glasgow outcome score $(1=$ death; $2-3=$ poor outcome; $4-5$ good outcome)

\section{Introduction}

Subarachnoid hemorrhage (SAH) has an overall incidence of $7-20 / 100000$ per year and accounts for $6 \%$ of total strokes. It affects a younger patient population and remains a serious condition with an overall mortality rate of approximately $45 \%$. About $15 \%$ of patients suffering from a SAH die before they reach medical attention. ${ }^{1}$ Given they survive the initial bleeding, $17-40 \%$ of the patients die or are often severely disabled due to sequelae of the bleeding such as intracerebral hematomas, hydrocephalus or delayed neurological deficits or ischemic infarctions (DCI) following cerebral vasospasm. ${ }^{2}$ The intensive care management of those patients remains challenging but offers an opportunity to improve outcome by reducing extracranial complications as well as secondary insults. ${ }^{1}$ Besides extracranial complications e.g. fever, cardiac and pulmonary abnormalities, cerebral vasospasm is the major cause of mortality and morbidity in SAH patients.

Treatment of cerebral vasospasm includes hypertension and hypervolemia therapy (HHT) or more invasive techniques like balloon angioplasty or intra-arterial application of vasodilators. The standard medical treatment of SAH patients includes oral application of nimodipine, a calcium channel blocker. Nimodipine is the only drug that has been proven beneficial for patient outcome, especially when cerebral vasospasm occurs. ${ }^{4}$ It is the most extensively studied and the only FDA approved drug for treatment of vasospasm. Like all calcium channel blockers it leads to vasodilation resulting in changes of systemic blood pressure. The intra venous (iv) application of nimodipine can result in significant and life threatening decrease of arterial blood pressure, bradycardia or even cardiovascular collapse leading to a boxed warning of the manufacturer to avoid iv application of the contents of nimodipine capsules. The IV form is not FDA approved in the US. In a FDA survey of more than 400000 patients taking oral nimodipine only $4,41 \%$ reported about side effects such as hypotension. But so far no life threatening effect on arterial blood pressure have been reported after correct oral application of nimodipine. In this paper we report about 4 cases of severe catechoalmine refractory hypotension after oral application of $60 \mathrm{mg}$ nimodipine in patients suffering from SAH.

\section{Materials and methods}

Vital signs and neurological status are closely monitored in SAH patients on our neurosurgical ICU. All SAH patients get a central venous line and continuous intra-arterial blood pressure monitoring. The mean arterial blood pressure (MAP) is always monitored in addition to systolic blood pressure. Before aneurysm is secured systolic blood pressure is kept $<160 \mathrm{mmHg}$ to avoid rebleeding if needed an external ventricular catheter with intracranial pressure (ICP) monitoring is also put in place to avoid hydroephalus Then cerebral perfusion pressure $(\mathrm{CPP}=\mathrm{MAP}-\mathrm{ICP})$ is calculated. The $\mathrm{CPP}$ is kept above $70 \mathrm{mmHg}$ according to our standard protocol. Patients receive a standard medication set including magnesium, fluids and oral nimodipine with a standard dosage of $6 \times 60 \mathrm{mg}$ per day. If vasospasm occurs patients are treated according to escalating standard operating procedures (SOP) including HHT, intubation and analgosedation as well as multimodal monitoring including brain tissue oxygenation $\left(\mathrm{P}_{b t} \mathrm{O}_{2}\right)$ and cerebral blood flow $(\mathrm{CBF})$. If $\mathrm{P}_{\mathrm{bt}} \mathrm{O}_{2}$ and $\mathrm{CBF}$ fall below 
normal values despite maximum HHT, intra-arterial nimodipine application is performed.

If systemic hypotension occurred after application of oral nimodipine it was counteracted by use of fluids and catecholamines, mainly norepinephrine. The following dosage of oral nimodipine was cancelled and after blood pressure stabilisationonly $30 \mathrm{mg}$ nimodipine were given. In case of patients needing norepinephrine above 0.5 $\mathrm{mg} / \mathrm{h}$ nimodipine was paused. We included all SAH patients in this paper who showed a significant prolonged systemic hypotension (MAP $<55 \mathrm{mmHg}$ ) after the first or second oral application of nimodipine refractory to fluids and catecholamines (norepinephrine and epinephrine) within 30 minutes, representing vasoplegia.

\section{Results}

A total of 4 patients overall met the above criteria and were included in this study. For patients demographics see Table 1.

Table I Patient demographics

\begin{tabular}{|c|c|c|c|c|c|c|c|c|}
\hline & Age & Gender & WFNS & Aneurysm & MAPI & MAP2 & Medication & GOS \\
\hline Patient I & 65 & $\mathrm{~m}$ & 2 & MCA & 48 & 78 & na & 4 \\
\hline Patient 2 & 35 & $f$ & 4 & ICA & 47 & 76 & na & 2 \\
\hline Patient 3 & 73 & $M$ & 5 & $\mathrm{ACoA}$ & 44 & 90 & Amlodipine $\beta$-blocker & 3 \\
\hline Patient 4 & 68 & $\mathrm{~F}$ & 3 & PCoA & 50 & 83 & Amlodipine $\beta$-blocker enalaprile & 4 \\
\hline
\end{tabular}

\section{Patient I}

Patient 1 was a 65 year old male with SAH WFNS 2 and an aneurysma of the left MCA. After angiography he was transferred to our ICU and aneurysm clipping was scheduled for the next day. He had no prior medication especially no anti-hypertensive treatment. After establishing a central venous line and the arterial pressure monitoring $60 \mathrm{mg}$ of Nimodipine were applied. About 10 minutes after a continuous decline in MAP was monitored and we tried to counteract by fluid and norepinephrine. Despite aggressive treatment MAP continued to stay low $(50 \mathrm{mmHg})$ and the patients awareness declined significantly. The MAP dropped even further to $48 \mathrm{mmHg}$. Despite adding epinephrine to the treatment, no sufficient increase in MAP could be achieved. Since application of vasopressin is not recommended in $\mathrm{SAH}$ patients due to its possible direct effects on cerebral vessels and decrease of CBF So we decided to try methylene blue application (200 $\mathrm{mg}$ over $30 \mathrm{~min}$ ). As early as 10 minutes after infusion start, MAP started to increase steadily and 20 minutes after application of methylene blue MAP was $78 \mathrm{mmHg}$. In parallel the patient improved and showed no neurological deficit at time of infusion end. Patient received no further dosage of nimodipine.

\section{Patient 2}

This patient was a 35 year old female with SAH WFNS 4 due to a ruptured ACI aneurysm. She was intubated and on controlled ventilation at time of hospital admission. She received standard arterial and venous lines and an external ventricular drainage. Nimodipine was given in standard dosage of $6 \times 60 \mathrm{mg}$ via nasogastric tube. After the first application only a slight and short decrease in MAP was seen. But shortly after the second dosage a rapid decline in blood pressure and thus in CPP was documented. Despite fluids and high doses of norepinephrin $(2.5 \mathrm{mg} / \mathrm{h})$ MAP stayed as low as 47 $\mathrm{mmHg}$ and CPP was $34 \mathrm{mmHg}$. Due to the need of improving CPP we decided to try methylene blue $300 \mathrm{mg} / 30 \mathrm{~min}$. The patient showed a rapid and steady increase in MAP shortly after infusion start and we were able to maintain sufficient MAP of $76 \mathrm{mmHg}$ afterwards. No second application of methylene blue was necessary and nimodipine application was stopped.

\section{Patient 3}

The third patient was a 73 year old male, intubated and ventilated on admission due to a SAH WFNS 5 after rupture of an aneurysma of the anterior communicating artery. The aneurysm was clipped in an emergency surgery. He was on antihypertensive treatment with amlodipine and B-blocker. After surgery he was admitted on our
ICU and received the standard medication set including nimodipine. After the first oral treatment MAP dropped significantly from 82 $\mathrm{mmHg}$ to $44 \mathrm{mmHg}$ and $\mathrm{CPP}$ was only $30 \mathrm{mmHg}$. After unsuccessful application of fluids and catecholamines as well as a bolus of 100 mg hydrocortisone leading to a slight elevation in MAP we applied methylene blue (200 mg over $30 \mathrm{~min}$ ). After the first application MAP could be raised to $68 \mathrm{mmHg}$ which was not sufficient since CPP was only $50 \mathrm{mmHg}$. After a second dose of methylene blue (100 mg over $15 \mathrm{~min}$ ) MAP was $90 \mathrm{mmHg}$ and we were able to keep it at this level by using norepinephrine $(1,0-1,5 \mathrm{mg} / \mathrm{h})$ and fluids.

\section{Patient 4}

Patient 4 was a 68 year old female with known hypertension being on B-blocker, enalaprile and amlodipine. She was brought in with SAH WFNS 3 due to an aneurysma of the posterior communicating artery. Due to increasing hydrocephalus her level of awareness deteriorated rapidly and she had to be intubated and mechanically ventilated. An external ventricular drainage was placed and the standard medication set was prescribed. After the first dosage of nimodipine we saw a rapid decline in MAP $(50 \mathrm{mmHg})$ and CPP. Despite fluid and catecholamine application no significant elevation in blood pressure could be achieved. After application of $200 \mathrm{mg}$ methylene blue twice over 30 min, we could establish a sufficient MAP of $83 \mathrm{mmHg}$ and were able to keep it stable with fluids and vasopressors.

\section{Discussion}

The management of patients suffering from SAH was initially directed at avoiding rebleeding, treatment of hydrocephalus and delayed ischemia neurological deficits following cerebral vasospasm. During the last years it has been revealed that SAH is a complex multisystem disease with a variety of medical complications that can contribute to poor outcome. ${ }^{5}$ This includes cardiac abnormalities $(20 \%)$ and $20-30 \%$ of SAH patients present with elevated cardiac enzymes and an increase in troponin I. ${ }^{1}$ Those patients are more prone to left ventricular dysfunction possibly leading to congestive heart failure and pulmonary edema- a symptom complex that resembles TakoTsubo syndrome, a transient cardiac dysfunction first described in Japan. ${ }^{6}$ Myocardial band necrosis, other neurocardiogenic injuries ${ }^{7}$ as well as cases of Tako-Tsubo syndrome are now reported after SAH. ${ }^{8,9}$ Also pulmonary edema mostly due to an alteration in vascular permeability can occur after SAH. Those patients are at risk for the so called ,neurogenic stunned myocardium“, a reversible impairment of the left ventricle. ${ }^{8}$ Latest theories evolve the catecholamine release as the main reason behind cardiopulmonary sequelae of $\mathrm{SAH} .{ }^{7,10}$ Catechoamine release can result in acute myocardial stress 
and contraction band necrosis possibly leading to left ventricular impairment. Besides these problems, transient arrhythmias are also frequent after SAH (25-75\%).

Despite these additional influences on outcome after SAH, cerebral vasospasm remains the major cause of mortality and morbidity. ${ }^{3} \mathrm{As}$ stated before, the only studied drug that has proven to improve outcome of SAH patients suffering from vasospasm and delayed neuronal ischemia remains nimodipine. Nimodipine (Figure 1) is a dihydropyridine (DHP) and exerts its action via blockage of L-type voltage dependent calcium channels and it is the only DHP that can pass the blood brain barrier due to the lipophilic formula. ${ }^{3}$ Common side effects of nimodipine as a DHPtype $\mathrm{ca}^{2+}$ channel blocker are constipation, nausea, headache, rash, edema, low blood pressure, drowsiness, dizziness and probably liver dysfunction. ${ }^{4}$ DHPs can also interact with the serum levels of a variety of drugs like carbamazepine or simvastatin, regulate aldosterone and cortisol secretion in endocrine cells of the adrenal cortex ${ }^{11}$ and are involved in the conduction of pacemaker signals in the heart. Their general mechanism of action is exerted via coupling on skeletal or smooth muscle as well as cardiac muscle cells. Nimodipine and other DHPs normally lead to decreased vascular resistance and thus lower arterial blood pressure by reducing the contraction of arterial smooth muscle cells causing vasodilation. This is normally followed by negative inotropic effects on the heart. ${ }^{12}$ In general DHPs have initially also a negative chronotrope effect but they do not decrease the responsiveness of the heart to input from the sympathetic nervous system. ${ }^{13}$ Thus due to baroreflexes sympathetic activity is often increased if systemic blood pressure drops significantly, leading to reflex tachycardia. This increases the oxygen demand of the heart and could be detrimental for patients suffering from ischemic symptoms. ${ }^{12}$

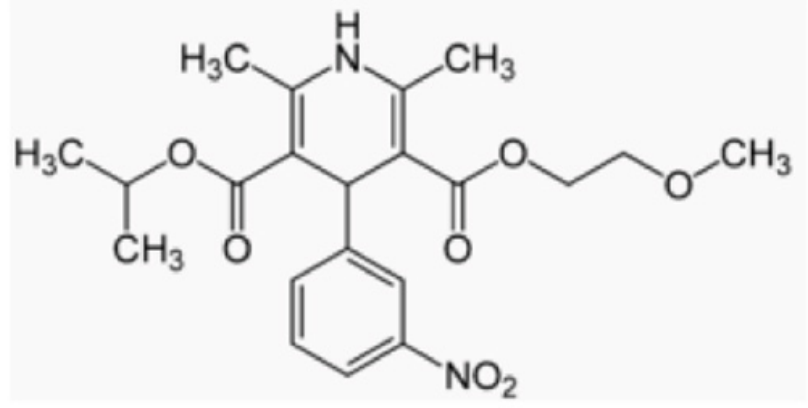

\section{Courtesy of Pharmaceuticals}

Figure I Nimodipine formula and systematic name.

Nimodipine systematic name: 2,6-Dimethyl-4-(3-nitrophenyl)- I,4dihydropyridine-3,5-dicarbonic acid-3-isopropylester-5-(2ethoxyethyl) ester.

A combination of the initial cardiopulmonary impairment after $\mathrm{SAH}$ and the effects nimodipine itself has on the heart and circulation could lead to significantly impaired left ventricular function and thus to the persistent drop in blood pressure seen in the 4 patients in our ICU. Ischemic changes and/or Tako-Tsubo syndrome might have led to decrease in contractility of the heart and lower output. This lower contractility might be the reason for an adverse reaction to fluids and catecholamine's. Unfortunately no transthoracal echo (TTE) was performed on the patients in this study so we don't know if any of the patients had signs of Tako-Tsubo or impaired left ventricular function, but we found elevated levels of troponin I in 3 of the 4 patients.

Another potentially accountable reason for the refractory hypotension could simply be an additive effect of prior medication.
Two patients were on amlodipine, another DHP, thus a simple addition of the vasodilator effects might be possible at least in those two patients.

Interestingly the form of hypotension we experienced in those 4 patients resembles the so called vasoplegic syndrome (VS).$^{14} \mathrm{VS}$ is often seen in patients after cardiac surgery, severe burns or septic shock. ${ }^{15}$ There is no standard definition of VS, but it includes significant hypotension, normal or even high cardiac output as well as low systemic vascular resistance (SVR) despite increasing requirement for catecholamines and fluids. ${ }^{16}$ These symptoms were found in our patients although we didn't have TEE to confirm normal cardiac output. Risk factors for developing VS include intravenous heparin, medication with long acting angiotensin converting enzyme (ACE) inhibitors and calcium channel blockers ${ }^{17-19}$ or systemic inflammatory response syndrome (SIRS). ${ }^{20}$ Also release of interleukines, which is seen after $\mathrm{SAH}$, can lead to vessel dilation by activation of cyclic guanosine monophosphate (cGMP). ${ }^{21}$ Thus it might be possible that nimodipine is a risk factor for developing VS after SAH under specific circumstances. These circumstances could be impaired left ventricular function, endothelial dysfunction etc. In combination with i.v. Heparin or premedication with another calcium channel blocker the risk for VS might even increase. Regardless of the initial ethiology, VS apparently represents a disbalance between synthesis and release of nitric oxide (NO). ${ }^{22}$ Endothelia nitric oxide synthase (eNOS) generates $\mathrm{NO}$ in endothelial and smooth muscle cells. NO is a extra- and intracellular signaling molecule that acts by inducing cGMP. cGMP relaxes smooth muscle cells and its most known function is vasodilation. ${ }^{22}$ Thus NO can exert potent vasodilative actions mostly by induction of loss of vascular sensitivity to catecholamines and myocardial depression. It has been described that SAH leads to systemic inflammatory reaction and is associated with increased oxidative stress in brain tissue. This correlates with a dysfunction of eNOS, normally leading to a depletion of NO and possibly endothelial dysfunction..$^{23,24}$ This could lead to an activation of eNOS to increase NO levels. In combination with increased endothelial permeability due to disturbed calcium signaling ${ }^{25}$ the eNOS activity could lead to increase in NO and cGMP followed by VS. Nimodipine possibly increases the disbalance of calcium signaling eventually adding NO release. In addition increased levels of Interleukines could add to the effect by activating cGMP.

When VS with no sufficient reaction to norepinephrine and fluids occurs, application of vasopressine and methylene blue might be the remaining possibilities. ${ }^{15,22}$ Since vasopressine might have a direct influence on cerebral vessel diameter and could decrease CBF it is not widely used in SAH patients to avoid additional vasospasm. ${ }^{26}$ Thus methylene blue remains as treatment option for patients suffering from VS. Methylene blue blocks accumulation of cGMP and thus reduces responsiveness of arterial vessels to cGMP dependent vasodilators like nitric oxide and Interleukines. ${ }^{15}$ Side effects of methylene blue consist in hypertension, headache, precordial pain and nausea as well as accumulation of met-hemoglobine. Since it is also a monoamine oxidase inhibitor if applied in high doses $(>5 \mathrm{mg} /$ $\mathrm{kg}$ ) it can precipitate serotonine toxicity, especially when the patient is on selective serotonine reuptake inhibitors. Thus methylene blue is not the drug of choice if low blood pressure occurs but remains the last resort if other measures fail to improve arterial blood pressure. A prophylactic application of methylene blue is not recommended due to possible side effects, unsufficient clinical data and because it leads to decrease in vessel diameter which might have an additional effect on cerebral vasospasm after SAH and can counteract the desired effect of nimodipine. 


\section{Conclusion}

In conclusion this is the first report about VS after oral application of nimodipine. The fact that only the application of methylene blue was able to counteract the effect of nimodipine in these patients suggests the involvement of endothelial dysfunction and increased $\mathrm{NO} / \mathrm{cGMP}$. Despite the fact that nimodipine is the only drug proven to have beneficial effects on outcome after cerebral vasospasm it should be applied with care and risk factors like VS should be taken into account.

We recommend to apply a strict regime in daily practice when it comes to application of oral nimodipine. This should include the check for additional medications that increase the risk of VS like $\mathrm{Ca}^{2+}$ antagonists and ACE-inhibitors or $\beta$-blockers. If patients are on this medication a test dosage of $15-30 \mathrm{mg}$ nimodipine seems a reasonable approach to avoid possible detrimental complications. However we recommend always to start with a test dosage of $30 \mathrm{mg}$ nimodipine irrespective of any known risk factors. We also approve the use of methylene blue if a prolonged hypotension refractory to norepinephrine occurs after oral nimodipine application.

\section{Acknowledgments}

This work was supported by the German Research Foundation (DFG) within the funding programme Open Access Publishing.

\section{Funding details}

None.

\section{Conflicts of interest}

Authors declare that there is no conflicts of interest.

\section{References}

1. Coppadoro A, Citerio G. Subarachnoid hemorrhage:an update for the intensivist. Minerva anestesiol. 2011;77(1):74-84.

2. Wu CT, Wong CS, Yeh CC, et al. Treatment of cerebral vasospasm after subarachnoid hemorrhage - a review. Acta Anaesthesiol Taiwan. 2004;42(4):215-222.

3. Dabus G, Nogueira RG. Current options for the management of aneurysmal subarachnoid hemorrhage-induced cerebral vasospasm:a comprehensive review of the literature. Interv Neurol. 2013;2(1):30-51.

4. Pickard JD, Murray GD, Illingworth R, et al. Effect of oral nimodipine on cerebral infarction and outcome after subarachnoid haemorrhage:British aneurysm nimodipine trial. BMJ. 1989;298(6674):636-642.

5. Wartenberg KE, Mayer SA. Medical complications after subarachnoid hemorrhage:new strategies for prevention and management. Curr Opin Crit Care. 2006;12(2):78-84.

6. Tsuchihashi K, Ueshima K, Uchida T, et al. Transient left ventricular apical ballooning without coronary artery stenosis:a novel heart syndrome mimicking acute myocardial infarction. Angina Pectoris-Myocardial Infarction Investigations in Japan. J Am Coll Cardiol. 2011;38(1):11-18.

7. Wybraniec MT, Mizia-Stec K, Krzych L. Neurocardiogenic injury in subarachnoid hemorrhage:A wide spectrum of catecholamin-mediated brain-heart interactions. Cardiol J. 2014;21(3):220-228.

8. Lee VH, Connolly HM, Fulgham JR, et al. Tako-tsubo cardiomyopathy in aneurysmal subarachnoid hemorrhage:an underappreciated ventricular dysfunction. J Neurosurg. 2006;105(2):264-270.
9. Zech N, Kieninger M, Seemann M, et al. Case report:Aneurysmatic subarachnoid hemorrhage - Complicated course due to coincidental manifestation of an inverted Tako-Tsubo-cardiomyopathy. Anasthesiol Intensivmed Notfallmed Schmerzther. 2014;49(7-8):428-434.

10. Macmillan CS, Grant IS, Andrews PJ. Pulmonary and cardiac sequelae of subarachnoid haemorrhage:time for active management? Intensive Care Med. 2002;28(8):1012-1023.

11. Kojima K, Kojima I, Rasmussen H. Dihydropyridine calcium agonist and antagonist effects on aldosterone secretion. Am J Physiol. 1984;247(5 Pt 1):E645-E650.

12. Low RI, Takeda P, Mason DT, et al. The effect of calcium channel blocking agents on cardiovascular function. American $J$ Cardiol. 1982;49(3):547-553.

13. Stone PH, Antman EM, Muller JE, et al. Calcium channel blocking agents in the treatment of cardiovascular disorders. Part II:Hemodynamic effects and clinical applications. Ann Intern Med. 1980;93(6):886-904.

14. Ozal E, Kuralay E, Yildirim V, et al. Preoperative methylene blue administration in patients at high risk for vasoplegic syndrome during cardiac surgery. Ann Thorac Surg. 2005;79(5):1615-1619.

15. Shanmugam G. Vasoplegic syndrome - the role of methylene blue. European journal of cardio-thoracic surgery. European Journal of Cardio-Thoracic Surgery. 2005;28(5):705-710.

16. Omar S, Zedan A, Nugent K. Cardiac Vasoplegia Syndrome:Pathophysiology, Risk Factors and Treatment. Am J Med Sci. 2014.

17. Mekontso Dessap A, Houel R, et al. Risk factors for postcardiopulmonary bypass vasoplegia in patients with preserved left ventricular function. Ann Thorac Surg. 2001;71(5):1428-1432.

18. Levin RL, Degrange MA, Bruno GF, et al. Methylene blue reduces mortality and morbidity in vasoplegic patients after cardiac surgery. Ann Thorac Surg. 2004;77(2):496-499.

19. Leyh RG, Kofidis T, Struber M, et al. Methylene blue:the drug of choice for catecholamine-refractory vasoplegia after cardiopulmonary bypass? J Thorac Cardiovasc surg. 2003;125(6):1426-1431.

20. Cremer J, Martin M, Redl H, et al. Systemic inflammatory response syndrome after cardiac operations. Ann Thorac Surg. 1996;61(6):1714-1720.

21. Beasley D, McGuiggin M. Interleukin 1 activates soluble guanylate cyclase in human vascular smooth muscle cells through a novel nitric oxide-independent pathway. J Exp Med. 1994;179(1):71-80.

22. Farina Junior JA, Celotto AC, da Silva MF, et al. Guanylate cyclase inhibition by methylene blue as an option in the treatment of vsoplegia after severe burn. Med Sci Monit. 2012;18(5):HY13-17.

23. Sabri M, Ai J, Marsden PA, et al. Simvastatin re-couples dysfunctional endothelial nitric oxide synthase in experimental subarachnoid hemorrhage. PloS One. 2011;6(2):e17062.

24. Sabri M, Ai J, Lass E, et al. Genetic elimination of eNOS reduces secondary complications of experimental subarachnoid hemorrhage. $J$ Cereb Blood Flow Metab. 2013;33(7):1008-1014.

25. Tiruppathi C, Minshall RD, Paria BC, et al. Role of $\mathrm{Ca} 2+$ signaling in the regulation of endothelial permeability. Vascul Pharmacol. 2002;39(4-5):173-185.

26. Bele S, Schebesch KM, Scheitzach J, et al. Vasopressin increases Cerebral Perfusion Pressure but not Cerebral Blood Flow in Neurosurgical Patients with Catecholamine-Refractory hypotension:A preliminary Evaluation unsing the Non-invasive Quantix ND in comparison to Literature. Journal of Anesthesia \& Critical Care:Open Access. 2013;1(3). 